(7\%) had systemic lupus erythematosus; 2 patients $(7 \%)$ had chronic rheumatoid arthritis; 9 patients $(30 \%)$ had other underlying risk factors, such as congenital or degenerative heart valve disease or coagulation problem; and 3 patients $(10 \%)$ had no risk factors. The majority of their patients underwent surgery for valve dysfunction.

When patients can tolerate cardiac surgery with cardiopulmonary bypass, indications for surgery are valve dysfunction, recurrent embolic events, ${ }^{4}$ and mobile vegetation. Removal of vegetation and repair of the valve seem to be feasible if the vegetation is small and localized. Prognosis after valve replacement or repair for nonbacterial thrombotic endocarditis is unknown but depends on the underlying cause.

Even if the previous femoropopliteal bypass was performed with the working diagnosis of Burger disease, it is unlikely because there was no arterial disease in the other leg. Because of the large size of the vegetation plus the possible delay of time between ischemic events, ${ }^{5}$ the patient's limb ischemia was thought to be arterial embolization caused by the vegetation. His noncoronary aortic leaflet was atrophic, which may be a factor in causing nonbacterial thrombotic endocarditis. Despite the patient's young age and good general condition, he should be monitored carefully for possible occult malignant disease.

\section{CONCLUSIONS}

Nonbacterial thrombotic endocarditis should be considered when a patient presents with recurrent embolization in the absence of a history of malignant disease, hypercoagulative state, or atrial fibrillation. Surgical removal of the vegetation is effective to prevent further embolization, and valve replacement or repair may be essential to correct valve dysfunction.

\section{References}

1. Borowski A, Ghodsizad A, Cohnen M, Gams E. Recurrent embolism in the course of marantic endocarditis. Ann Thorac Surg. 2005;79:2145-7.

2. Patrick WE, William DE, Henry DT, Robert DM, Kenton JZ. Surgical pathology of nonbacterial thrombotic endocarditis in 30 patients, 1985-2000. Mayo Clin Proc. 2001;76:1204-12.

3. Kardaras FG, Kardara DF, Rotntoglani DP, Sioras EP, Christopoulou-Cokkinou V, Lolas CT, et al. Acute aortic regurgitation caused by non-bacterial thrombotic endocarditis. Eur Heart J. 1995;16:1152-4.

4. Rabinstein AA, Giavanelli C, Ricci M, Romano JG, Koch S, Forteza AM. Surgical treatment of nonbacterial thrombotic endocarditis presenting stroke. J Neurol. 2005;252:352-5.

5. Callander N, Rapaport SI. Trousseau's syndrome. West J Med. 1993;158: 364-71.

\title{
Multiple papillary fibroelastoma with quadricuspid aortic valve
}

Reiji Hattori, MD, PhD, ${ }^{\text {a }}$ Chihiro Oishi, MD, Junji Iwasaka, MD, PhD, ${ }^{\mathrm{b}}$ Toshiji Iwasaka, MD, PhD, ${ }^{\mathrm{b}}$ Takayuki Okada, MD,

Hiroyuki Johno, MD, ${ }^{\mathrm{a}}$ Chiharu Enoki, MD, ${ }^{\mathrm{a}}$ Tomohiko Sumida, MD, ${ }^{\mathrm{a}}$ Yoshihisa Nakao, MD, PhD, ${ }^{\mathrm{a}}$ and

Hiroji Imamura, MD, PhD, ${ }^{a}$ Hirakata, Osaka, Japan

Quadricuspid aortic valve (QAV) is a rare congenital anomaly that is one of the causes of aortic valve insufficiency, which often requires aortic valve replacement. Cardiac papillary fibroelastoma $(\mathrm{CPF})$ is also a rare benign cardiac tumor, and there are reports of rare cases of multiple occurrences. We report our experience of successful surgery for a previously unreported condition in a patient with a QAV who had a narrow aortic annulus associated with complications of multiple CPF.

From the Department of Thoracic and Cardiovascular Surgery ${ }^{\mathrm{a}}$ and Division of Cardiology, Second Department of Medicine, ${ }^{\mathrm{b}}$ Kansai Medical University, Hirakata, Osaka, Japan.

Received for publication March 21, 2008; accepted for publication April 20, 2008.

Address for reprints: Reiji Hattori, MD, PhD, Department of Thoracic and Cardiovascular Surgery, Kansai Medical University, 2-3-1, Shinmachi, Hirakata, Osaka, 573-

1191, Japan (E-mail: hattori@hirakata.kmu.ac.jp).

J Thorac Cardiovasc Surg 2009; 137:1280-2

$0022-5223 / \$ 36.00$

Copyright (c) 2009 by The American Association for Thoracic Surgery doi:10.1016/j.jtcvs.2008.04.015

\section{CLINICAL SUMMARY}

A 71-year-old woman underwent a transthoracic echocardiogram as part of a detailed examination for the treatment of peripheral arterial disease, and mobile masses were incidentally found that were attached to the aortic valve. In addition, the aortic valve was a QAV with severe aortic regurgitation, and various sizes of mobile masses were attached to each of the 4 cusps detected by a transesophageal echocardiogram (Figure 1). Moreover, similar mobile masses were also observed in the left ventricular outflow tract (LVOT) and mitral chordae. In November of 2007, the patient underwent an urgent operation. The aortic valve was observed through an oblique aortotomy using a standard technique of cardiopulmonary bypass under cardiac arrest obtained with blood cardioplegia. Various sizes of mobile tumors were attached to each of the 4 cusps on the ventricular side. The aortic valve was carefully excised with the attached tumors. Through observations of the removed aortic valve placed in water, we found that 

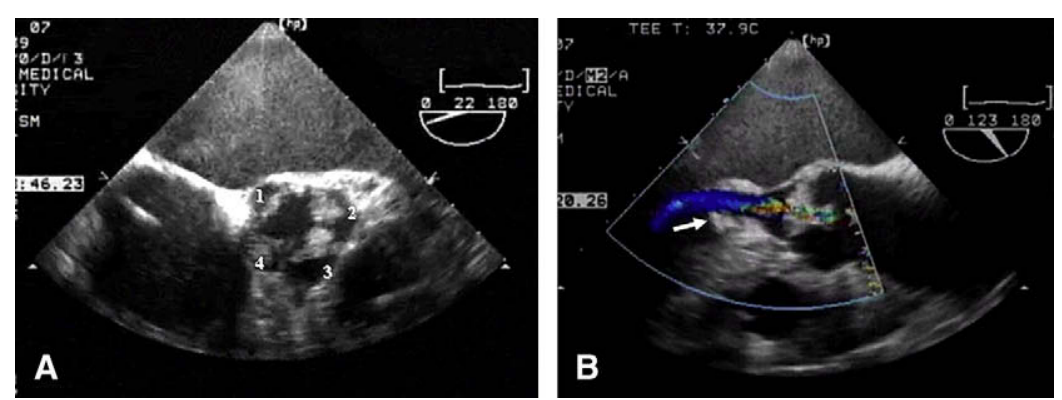

FIGURE 1. Transesophageal echocardiogram. A, QAV with various sizes of mobile masses. B, Severe aortic regurgitation and mobile masse on LVOT (arrow). The portions to which the CPF were attached in the LVOT and mitral chordae were portions that were subjected to the aortic regurgitation jet.
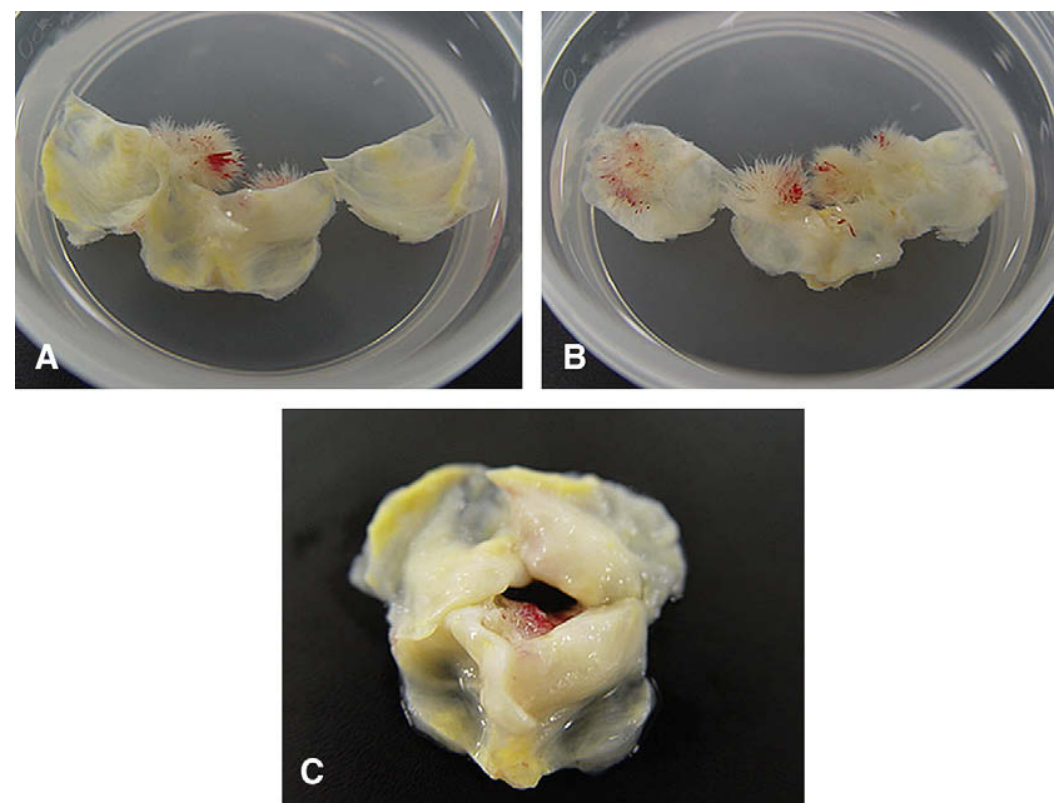

FIGURE 2. Gross specimen of the QAV with tumors. A, No tumors are observed on surface of the aorta side. B, Various sizes of sea anemone-like tumors are attached to all 4 cusps of the ventricular side. C, QAV showing fibrotic change.

all of the tumors were sea anemone-like tumors with multiple papillary fronds (Figure 2). Similar tumors in the LVOT and mitral chordae were also excised. Because the aortic annulus was diminutive, a $17-\mathrm{mm}$ Sorin Bicarbon Slimline (Sorin Biomedica Cardio S.r.l., Saluggia, Italy) was replaced in the supra-annular position. The tumor was diagnosed to be a CPF on the basis of a histologic examination. The patient successfully recovered, and transthoracic echocardiogram showed no patient-prosthesis mismatch with an indexed effective orifice area of 1.05 .

\section{DISCUSSION}

In a QAV, the additional smaller cusp causes unequal distribution of mechanical stress and can lead to the development of progressive cusp fibrosis and calcification at an earlier stage. ${ }^{1}$ The patient's QAV consisted of 2 equally large cusps and 2 equally small cusps and was classified as Hurwitz-Roberts classification type C. ${ }^{2}$ At the same time, it has been pointed out that CPF develops at sites where damage and stress are incurred. ${ }^{3}$ More specifically, it is believed that QAV may create conditions that make the development of CPF easier than in a normal valve. The portions to which the CPF were attached in the LVOT and mitral chordae were subjected to the aortic regurgitation jet, and these portions also exhibited mechanical stress. Furthermore, this patient also had a narrow aortic annulus. There has been only 1 reported case of QAV with a narrow aortic annulus in the past, and it is hypothesized that hypoplasia of the aortic annulus is caused by aAV that may be part of a more complex pathologic condition of the LVOT. ${ }^{1}$ It is believed that more stress was applied to the aortic valve because of 
the narrow aortic annulus and that the development of CPF may have been promoted.

In this case, endocarditis was initially suspected because of vegetation-like mobile masses that were found in the aortic valve on the transesophageal echocardiogram, but infection was negative in all other examination findings. We determined before surgery that these masses were most likely cardiac tumors. Mild exertional dyspnea was the only clinical symptom, but it is known that mobile tumors attached to an aortic valve can cause serious complications such as thromboembolism and myocardial ischemia, ${ }^{4}$ and we decided that a surgical excision was necessary.

QAV with a narrow aortic annulus presenting with the development of multiple CPFs is a rare complication. There have been no similar cases reported in the literature. However, according to the characteristics of each pathologic condition, there is a possibility that CPF may develop with QAV.

\section{References}

1. Croccia MG, Chiaramonti F, Pratali S, Scioti G, Guarracino F, Bortolotti U. Quadricuspid aortic valve associated with hypoplastic aortic annulus. J Thorac Cardiovasc Surg. 2007;134:1061-2.

2. Hurwitz LE, Roberts WC. Quadricuspid semilunar valves. Am J Cardiol. 1973;31:623-6.

3. Klarich KW, Enriquez-Sarano M, Gura GM, Edwards WD, Tajik AJ, Seward JB. Papillary fibroelastoma: echocardiographic characteristics for diagnosis and pathologic correlation. J Am Coll Cardiol. 1997;30:784-90.

4. Jobic Y, Etienne Y, Quintin-Roué I, Dewilde J, Cornec P, Gilard M, et al. Left ventricular papillary fibroelastoma: two-dimensional echocardiographic detection and surgical resection. J Am Soc Echocardiogr. 1995;8:756-8.

\section{Pulmonary venous obstruction in a patient with Marfan syndrome: Rare presentation of an expanding dissecting descending thoracic aortic aneurysm with annuloaortic ectasia}

Yoshikatsu Saiki, MD, PhD, Shunsuke Kawamoto, MD, PhD, Junetsu Akasaka, MD, PhD, Naotaka Motoyoshi, MD, PhD, Sadahiro Sai, MD, PhD, and Koichi Tabayashi, MD, PhD, Sendai, Japan

Pulmonary venous obstruction (PVO) is an extremely rare condition in patients with acquired cardiovascular disease. We describe the case of a patient with chronic dissecting descending thoracic aortic aneurysm and annuloaortic ectasia associated with Marfan syndrome in whom PVO developed. The patient underwent successful emergency single-stage repair of the extensive thoracic aortic aneurysm.

\section{CLINICAL SUMMARY}

A 15-year-old female patient with Marfan syndrome had been followed up at a local hospital for annuloaortic ectasia with mild aortic insufficiency. A type B acute aortic dissection developed and was managed conservatively. Four months later, the patient had sudden onset of severe back pain, and computed tomographic (CT) scans revealed expansion of the dissecting descending thoracic aorta. She

From the Department of Cardiovascular Surgery, Graduate School of Medicine, Tohoku University, Sendai, Japan.

Received for publication Dec 23, 2007; accepted for publication Jan 12, 2008.

Address for reprints: Yoshikatsu Saiki, MD, Department of Cardiovascular Surgery, Graduate School of Medicine, Tohoku University, Seiryomachi, Aoba-ku, Sendai,

Japan 980-8574 (E-mail: ysaiki@mail.tains.tohoku.ac.jp).

J Thorac Cardiovasc Surg 2009;137:1282-4

0022-5223/\$36.00

Copyright $@ 2009$ by The American Association for Thoracic Surgery doi:10.1016/j.jtcvs.2008.01.043 was admitted to our intensive care unit because rupture of the thoracic aorta appeared impending. Sixteen hours after admission, large amounts of foamy sputum were observed. Repeated chest radiographs revealed pulmonary venous congestion, specifically on the right side (Figure 1, A). A transthoracic echocardiogram revealed a moderate degree of aortic regurgitation and turbulent right pulmonary venous flow. On the basis of the CT scans, the PVO on the right side was attributed to compression by three anatomic components: the expanding dissecting descending thoracic aortic aneurysm, the annuloaortic ectasia, and the deformed thorax (Figure 1, B). Emergency surgery was undertaken to repair the extensive disease of the thoracic aorta and to resolve the PVO.

The patient was placed in a semidecubitus position with her left side facing up. Prosthetic vascular grafts $(6 \mathrm{~mm})$ were anastomosed to the sides of the right subclavian, left axillary, and left femoral arteries, for arterial inflow. Bilateral anterior thoracotomy through the fourth intercostal space with limited incision on the right side, transverse sternotomy, transection of the fifth and sixth ribs, and further extension of the sixth intercostal incision were used to expose the entire thoracic aorta and heart (Figure 2, A). Aortic root replacement with a composite graft with a bileaflet mechanical valve was performed under conditions of hypothermic 